\title{
Acromial approach for treating glenoid fractures: A report of two cases and a literature review
}

\author{
RONGGUANG AO, BAOQING YU, JIFEI SHI, ZEXIANG LI and YALONG ZHU \\ Department of Orthopedics, Shanghai Pudong Hospital, Fudan University Pudong Medical Center, \\ Shanghai 201399, P.R. China
}

Received September 30, 2014; Accepted August 11, 2015

DOI: $10.3892 /$ etm.2015.2717

\begin{abstract}
Surgery is usually recommended for displaced glenoid fractures, with open reduction and internal fixation as the standard operative treatment. Three approaches have been recommended in the reduction of glenoid fractures: Anterior, posterior and combined; however, a traditional approach may be difficult due to a high position or a comminuted Ideberg type III fracture. The combined approach results in a longer incision and more soft tissue injury when associated with an acromial fracture. The present study describes two complicated glenoid fractures: One case was a comminuted Ideberg type III fracture associated with an Ogawa type II acromial fracture; the second case was an Ideberg type IV fracture with associated superior shoulder suspensory complex injuries. In these cases, the acromial approach was modified to achieve satisfactory exposure and fixation of the complicated fracture. After a 1-year follow-up, the patients had a satisfactory outcome.
\end{abstract}

\section{Introduction}

Ideberg type III and type IV fractures are unique, involving the superior glenoid cavity, and the fracture line commonly extends to the medial area of the coracoids (1). Surgical treatment is usually recommended for displaced Ideberg type III fractures (2). Three approaches have been reported for such fractures: Anterior $(3,4)$, posterior $(5,6)$ and combined $(7)$. Considerable difficulties are associated with the exposure and fixation of a glenoid fracture fragment around the scapular notch using these three traditional approaches due to the acromion. To the best of our knowledge, the treatment of Ideberg type III glenoid fractures using an acromial approach has not been reported in the literature. The present study describes the

Correspondence to: Dr Baoqing Yu, Department of Orthopedics, Shanghai Pudong Hospital, Fudan University Pudong Medical Center, 2800 Gongwei Road, Huinan Town, Pudong, Shanghai 201399, P.R. China

E-mail: doctorybq@163.com

Key words: acromion, approach, Ideberg type III fractures, case, review treatment of two cases of complicated glenoid fractures using the acromial approach and presents a review of the literature on the scapular glenoid fracture.

\section{Case report}

Case 1. A 30-year-old man complained of pain in the left shoulder following a direct blow from an object weighing $\sim 100 \mathrm{~kg}$. Written informed consent was obtained. The plain radiography showed a glenoid fracture associated with an Ogawa type II acromial fracture (Fig. 1). Three-dimensional computed tomography indicated a comminuted glenoid fracture (Ideberg type III) and a displaced Ogawa type II acromial fracture (Fig. 2). The comminuted glenoid fracture consisted of two fragments; the anterior fragment extended to the medial area of the coracoid, while the posterior fragment was located around the scapular notch. The patient was admitted to our department at the Shanghai Pudong Hospital (Shanghai, China) for surgery 3 days after the injury. The glenoid fracture was addressed first using open reduction via the acromial approach, pulling the acromial fracture segment and fixing it using three screws. The acromial fracture was then fixed using a reconstructed locking plate (Fig. 3).

The patient was followed-up for 13 months. No wound or axillary nerve complications were noted. Fracture union was achieved 8 weeks after the surgery. At the final follow-up, the patient achieved satisfactory shoulder function. The shoulder abduction, forward flexion and external rotation were 135, 150 and $50^{\circ}$, respectively, which were 25,15 and $17^{\circ}$ less than the corresponding movement ranges in the contralateral shoulder, respectively. Internal rotation of the operative and contralateral shoulders was at thoracic levels T6 and T4, respectively. The Constant-Murley, University of California, Los Angeles (UCLA) (8) and Disabilities of Arm, Shoulder and Hand (DASH) (9) scores were 84, 32 and 15 points, respectively.

Case 2. A 58-year-old man was injured in a motorbike accident. Written informed consent was obtained. The patient suffered a displaced distal clavicular fracture, multiple-rib fracture and a scapular fracture involving a displaced glenoid fracture (Ideberg type IV) (Fig. 4).

The patient was transferred to the Department of Thoracic Surgery at the Shanghai Pudong Hospital. Following open 
reduction and fixation of the fractured ribs, the patient was admitted to our department at the Shanghai Pudong Hospital for surgery 10 days post-injury. The surgical procedure was similar to that in case 1 . First, the glenoid fracture was addressed using an open reduction via the acromial approach, pulling the acromial fracture segment and fixing it using a locking plate. The acromial and scapular fractures were then fixed using locking plates, prior to the fixation of the distal clavicular fracture using a locking plate (Fig. 5).

The patient was followed-up for 13.5 months. There were no wound or axillary nerve complications. Fracture union was achieved 9 weeks after the surgery. At the final follow-up, the patient achieved satisfactory shoulder function. The shoulder abduction, forward flexion and external rotation were 130,148 and $55^{\circ}$, respectively, which were 28,18 and $15^{\circ}$ less than the corresponding movement ranges in the contralateral shoulder, respectively. Internal rotation of the operative and contralateral shoulders was at thoracic levels T6.2 and T4, respectively. The Constant-Murley, UCLA and DASH scores were 81, 29 and 18 points, respectively.

\section{Surgical techniques}

Case 1. The patient was placed in the lateral position with the affected side up and the forearm was draped free so that it could be mobilized during the procedure. The position of the incision was marked, and the incision was performed from the posterior acromion along the middle length of the scapular spine. The acromion was readily exposed from its subcutaneous position, and the deltoid muscle was identified, detached from the posterior of the scapular spine and retracted inferiorly. The trapezius muscle was identified, detached from the anterior of the scapular spine and retracted anteriorly. Following the removal of the anterior and posterior soft tissue that was adhered to the acromion, the acromial fracture fragment was raised laterally, the affected shoulder was abducted $90^{\circ}$ and the supraspinatus muscle tendon was pulled superiorly together with the acromial fragment. The two glenoid fracture fragments were visualized. First, the anterior part combined with the coracoids was reduced manually using clamps and fixed temporarily using K-wire. The posterior part around the scapular notch was then reduced and fixed using K-wire. Two screws were used to fix the anterior fracture section and one for the posterior section. Finally, the acromial fracture was reduced and readily fixed using a locking plate. It should be noted that the cut supraspinatus muscle tendon should be sutured carefully prior to managing the acromial fracture. At the end of each step, C-arm fluoroscopy was performed to monitor the fracture reduction and the length of the screws in the standard anteroposterior and axillary views of the scapula.

Case 2. The patient was positioned as in case 1, again with the forearm draped free. The position of the incision was marked, and the incision was performed from the posterior acromion along the entire length of the scapular spine, curving distally along the medial border of the scapula and extending proximally to the distal clavicle. The deltoid muscle was identified, detached from the posterior of the scapular spine and retracted inferiorly. The trapezius muscle was identified, detached from the anterior of the scapular spine and retracted anteriorly. Subsequently, the infraspinatus was visualized and

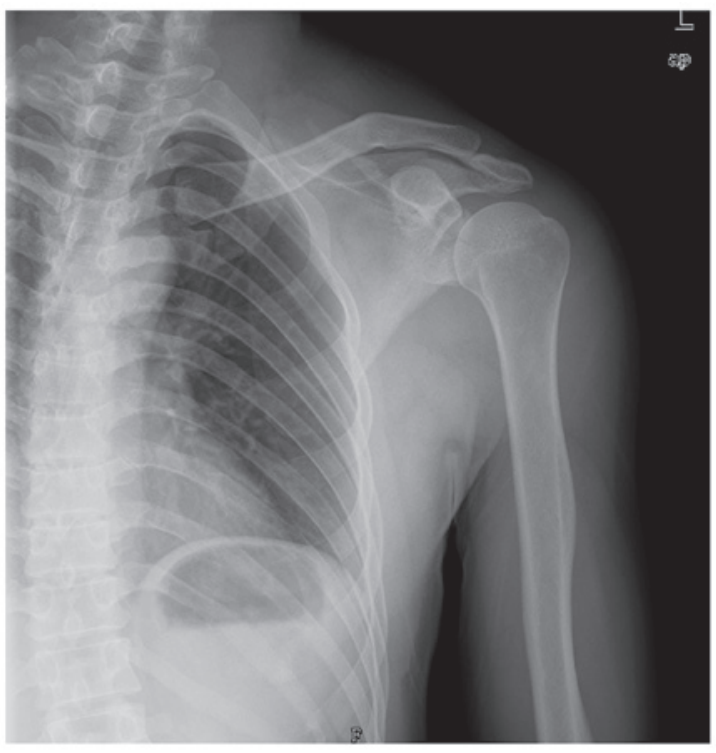

Figure 1. Preoperative X-ray showed the left glenoid fracture with an acromial fracture.
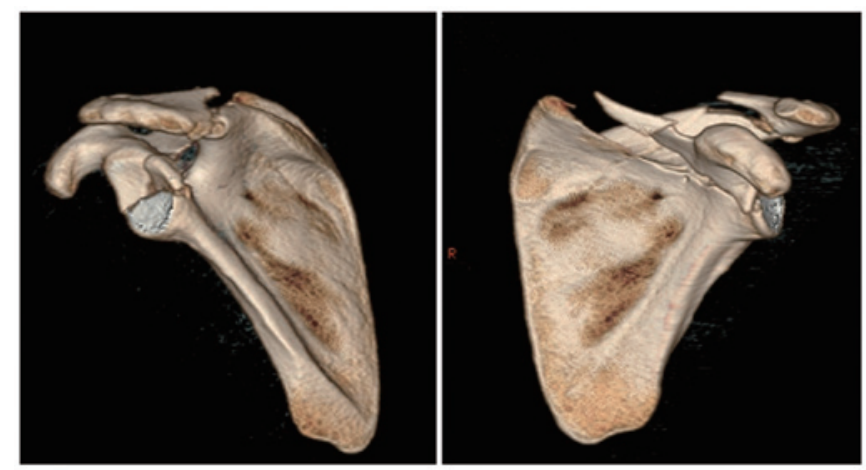

Figure 2. Preoperative three-dimensional reconstruction showed the comminuted Ideberg type III glenoid fracture, with the posterior-superior fragment located around the scapular notch.

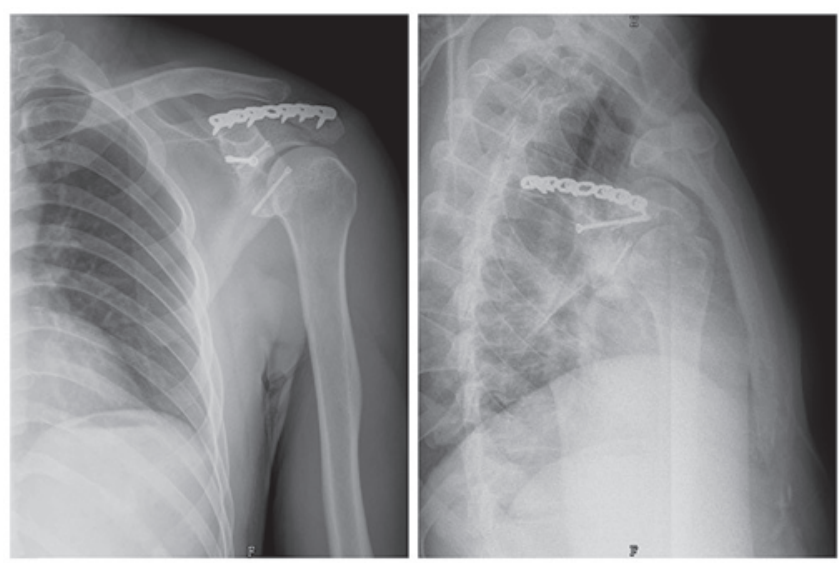

Figure 3. Postoperative X-ray.

bluntly separated inferiorly to access the scapular spine and body fracture fragments. The acromial basilar fracture line was exposed. Following the removal of the anterior and posterior soft tissue that was adhered to the acromion and part of 

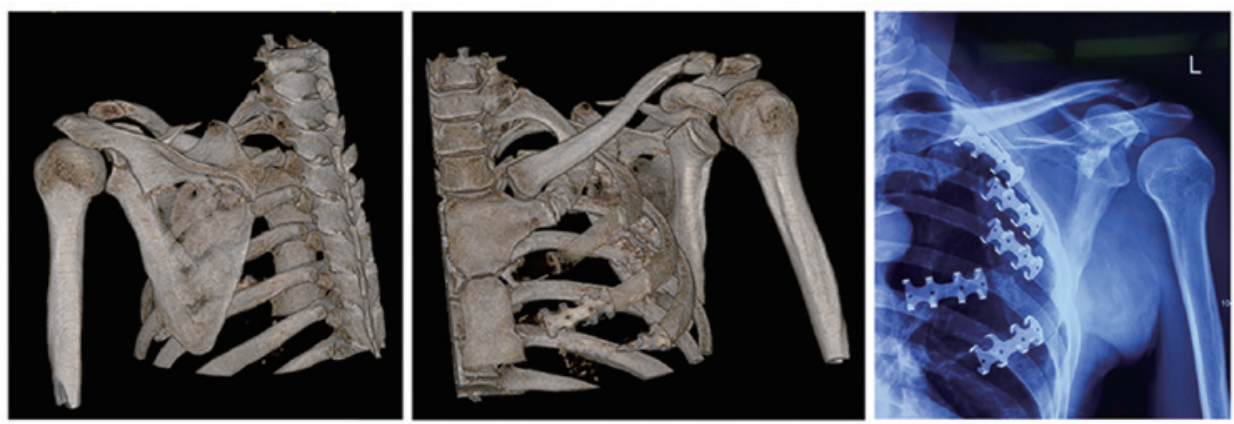

Figure 4. Preoperative three-dimensional reconstruction and X-ray showed the Ideberg type IV glenoid fracture associated with a displaced distal clavicular fracture. Multiple rib fractures had been treated surgically.

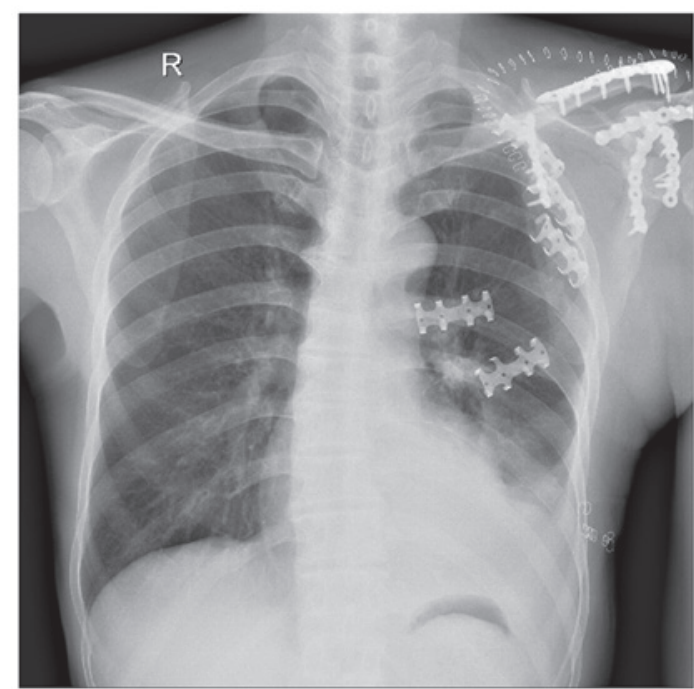

Figure 5. Postoperative X-ray.

the scapular spine, the acromial fracture fragment was raised laterally, the affected shoulder was abducted $90^{\circ}$ and the supraspinatus muscle tendon was pulled superiorly together with the acromial fragment. The glenoid fracture was clearly exposed, reduced and fixed using a locking plate. The extra-articular regions of the scapular and distal clavicular fractures were then monitored, reduced and fixed using a locking plate.

\section{Discussion}

Scapular fractures are uncommon injuries, accounting for $1 \%$ of all fractures; $10 \%$ of these involve the glenoid cavity (10). Direct violent trauma is the normal cause of scapular fractures. A direct violent force applied laterally to the proximal humerus, which drives the humeral head into the glenoid fossa, can lead to a glenoid fracture. When the force of the humeral head is directed slightly superiorly, it can result in an Ideberg type III and IV glenoid fracture, leading to a transverse fracture of the fossa that exits along the superior border of the scapula. When the force is sufficiently large, it will disrupt the superior shoulder suspensory complex (SSSC), including the acromion, acromioclavicular joint and distal clavicle (11).

The indications for surgical treatment include intra-articular fractures with a $>5-\mathrm{mm}$ articular displacement, step-off and instability $(12,13)$. A further operative indication for scapular fractures is a double disruption of the SSSC. The SSSC, which consists of the glenoid, coracoid, acromion, distal clavicle, coracoclavicular ligaments and acromioclavicular ligaments, secures the upper extremity to the axial skeleton (14). While single disruptions of the SSSC are generally stable, instability can result when the SSSC is disrupted in two different locations (double disruption). According to Goss (14), open reduction and internal fixation (ORIF) are indicated for SSSC double disruptions that are accompanied by significant displacement, as these may lead to delayed union, malunion or nonunion, as well as long-term functional deficits. In a study by Qin et al (11), 9 patients (1 further patient was lost to follow-up) with Ideberg type III fractures associated with SSSC injuries were successfully treated for glenoid fractures and SSSC injuries.

Surgical treatment for the glenoid fracture includes ORIF and a percutaneous arthroscopy-based procedure. Although the latter method is minimally invasive and associated with a reduction in approach-related morbidity, and thus a more rapid return to function, it is demanding using the current technology. In particular, correct screw placement remains difficult, and general experience in the surgical management is limited $(15,16)$. ORIF is the standard surgical treatment for the glenoid fracture, even when approach-related complications, including persistent pain, reduced range of motion and weakened maximum isokinetic muscle strength, have to be considered $(17,18)$.

Three approaches have been recommended to reduce glenoid fractures: Anterior $(3,4)$, posterior $(5,6)$ and combined (7). The anterior approach is the most common method for the Ideberg type III fracture due to the satisfactory exposure, including of the intra- and extra-articular components; however, the disadvantage of this approach is the extensive soft tissue dissection that is required, including resection of the subscapularis and its capsule, and the elevation of the subscapularis from the ventral aspect of the scapular body, which may affect the internal shoulder rotation. In addition, fractures around the scapular notch are difficult to expose and fix using the anterior approach (11).

The posterior approach is frequently used for the treatment of scapular body, scapular neck and glenoid fractures with an associated posterior articular component (19). As a result of the positioning of the acromion, it is impossible to expose and fix the posterior-superior articular component using the posterior approach. 
Currently, there is no anatomical research on a visible glenoid approach for the reduction and fixation of a glenoid fracture fragment located around the scapular notch; therefore, the approach can only be selected based on a surgeon's experience and understanding of the shoulder anatomy. The basilar region of the coracoid is located anterior-superior to the glenoid, and the Ideberg type III fracture line often extends to the medial area of the coracoid. In addition, the acromion is located upon the posterior-superior region of the glenoid, and the component around the scapular notch is deep and therefore difficult to expose and fix. To the best of our knowledge, there is no relevant anatomical research on the positional association between the acromion and the glenoid; therefore, it is difficult to expose and fix an Ideberg type III and IV fracture with the fragment around the scapular notch.

Ideberg type III and IV glenoid fractures are often associated with SSSC injuries, including acromial, acromioclavicular joint and distal clavicular fractures. These injuries can be monitored under direct vision using the anterior approach, extending the incision superiorly; when associated with acromial fracture, the additional incision would be used, therefore, additional injuries are created.

In principle, an Ideberg type III and IV fracture associated with an acromial fracture could be exposed and fixed by pulling the middle or basilar acromial fragment superiorly and pushing the supraspinatus muscle tendon. The so-called acromial approach would require a smaller incision and cause less soft tissue injury compared with the combined approach.

There is debate as to the existence of a superior approach, causing less soft tissue injury and with satisfactory exposure, for the management of an Ideberg type III fracture around the scapular notch or associated with an acromial fracture. In the two cases reported in the present study, we believed that the acromial approach would afford a more visible operating space for the glenoid around the scapular notch, enable easier exposure and fixation of the fracture than the anterior approach and produce less soft tissue injury than the combined approach. Furthermore, it was considered that raising the supraspinatus muscle tendon instead of cutting it would cause no significant limitation to the abduction of the affected shoulder. It should be noted that the acromial fracture line should be located in the middle of or medial to the entire acromion. When the line is located around the extremitas acromialis, the medial region of the acromion can restrict the exposure and reduction. Management of the supraspinatus muscle tendon is important, as even partial cutting of the tendon is likely to lead to a significant limitation in the abduction. Based on experience, we abducted the affected shoulder to relax the supraspinatus muscle tendon, allowing the successful elevation of the tendon to expose the glenoid fracture fragments around the scapular notch and coracoid.

Surgical treatment of glenoid fractures is particularly challenging with respect to the exposure of the fracture. The glenoid fragment around the scapular notch is extremely difficult to expose due to the acromion. Coincidentally, the two cases in the present study were both associated with an acromial fracture; therefore, the supraspinatus muscle tendon was elevated in each case, satisfactorily exposing the glenoid fracture fragments around the scapular notch and coracoid.
Following effective functional training, both patients achieved good shoulder function. In the future, we aim to perform anatomical research on the exposure of the glenoid fragment and to determine the visible area of the glenoid fragment using the anterior and posterior approaches, as well as the acromial approach. The acromial approach may provide an alternative method for Ideberg type III glenoid fractures around the scapular notch or associated with an acromial fracture.

\section{Acknowledgements}

This study was funded by the Key Disciplines Group Construction Project of Pudong Health Bureau of Shanghai (grant no. PWZxq2014-03).

\section{References}

1. Ideberg R, Grevsten S and Larsson S: Epidemiology of scapular fractures. Incidence and classification of 338 fractures. Acta Orthop Scand 66: 395-397, 1995.

2. Owens BD and Goss TP: Surgical approaches for glenoid fractures. Tech Shoulder Elbow Surg 5: 103-115, 2004.

3. Cole PA, Gauger EM and Schroder LK: Management of scapular fractures. J Am Acad Orthop Surg 20: 130-141, 2012.

4. Anavian J, Gauger EM, Schroder LK, Wijdicks CA and Cole PA: Surgical and functional outcomes after operative management of complex and displaced intra-articular glenoid fractures. J Bone Joint Surg Am 94: 645-653, 2012.

5. Schandelmaier P, Blauth M, Schneider C and Krettek C: Fractures of the glenoid treated by operation. A 5- to 23-year follow-up of 22 cases. J Bone Joint Surg Br. 84: 173-177, 2002.

6. Adam FF: Surgical treatment of displaced fractures of the glenoid cavity. Int Orthop 26: 150-153, 2002.

7. Gramstad GD and Marra G: Treatment of glenoid fractures. Tech Shoulder Elbow Surg 3: 102-110, 2002.

8. Amstutz HC, Sew Hoy AL and Clarke IC: UCLA anatomic total shoulder arthroplasty. Clin Orthop Relat Res 155: 7-20, 1981.

9. Longo UG, Vasta S, Maffulli N and Denaro V: Scoring systems for the functional assessment of patients with rotator cuff pathology. Sports Med Arthrosc 19: 310-320, 2011.

10. Zlowodzki M, Bhandari M, Zelle BA, Kregor PJ and Cole PA: Treatment of scapula fractures: Systematic review of 520 fractures in 22 case series. J Orthop Trauma 20: 230-233, 2006.

11. Qin H, Hu CZ, Zhang XL, Shen LX, Xue ZC and An ZQ: Surgical treatment of Ideberg type III glenoid fractures with associated superior shoulder suspensory complex injury. Orthopedics 36: e1244-e1250, 2013.

12. Bauer G, Fleischmann W and Dussler E: Displaced scapular fractures: Indication and long-term results of open reduction and internal fixation. Arch Orthop Trauma Surg 114: 215-219, 1995.

13. Soslowsky LJ, Flatow EL, Bigliani LU and Mow VC: Articular geometry of the glenohumeral joint. Clin Orthop Relat Res: 181-190, 1992.

14. Goss TP: The scapula: Coracoid, acromial, and avulsion fractures. Am J Orthop (Belle Mead NJ) 25: 106-115, 1996.

15. Yang HB, Wang D and He XJ: Arthroscopic-assisted reduction and percutaneous cannulated screw fixation for Ideberg type III glenoid fractures: A minimum 2-year follow-up of 18 cases. Am J Sports Med 39: 1923-1928, 2011.

16. Gras F, Marintschev I, Aurich M, Rausch S, Klos K and Hofmann GO: Percutaneous navigated screw fixation of glenoid fractures. Arch Orthop Trauma Surg 133: 627-633, 2013.

17. Raiss P, Baumann F, Akbar M, Rickert M and Loew M: Open screw fixation of large anterior glenoid rim fractures: Mid- and long-term results in 29 patients. Knee Surg Sports Traumatol Arthrosc 17: 195-203, 2009.

18. Scheibel M, Magosch P, Lichtenberg S and Habermeyer P: Open reconstruction of anterior glenoid rim fractures. Knee Surg Sports Traumatol Arthrosc 12: 568-573, 2004.

19. Gauger EM and Cole PA: Surgical technique: A minimally invasive approach to scapula neck and body fractures. Clin Orthop Relat Res 469: 3390-3399, 2011. 